\title{
My amazing experience
}

@ $\mathrm{n}$ my junior year at high school I was offered to do a physics project instead of the ordinary exam. The idea of conducting real scientific research intrigued me, so I started working right away with Haggai Landa from the University of Tel Aviv as my guide. We performed various ion-trapping simulations of equally charged cold ions in Multipole Paul traps. We studied their structures, the configurations they created (such as crystals of one or more rings), defects, and the vibrations they performed around the minimum of their potential energy. Moreover, I visited Roee Ozeri's laboratory at the Weizmann Institute of Science, the only place in Israel where experiments with actual ion traps are being conducted. Little did I know that later on a Nobel Prize would be given in this very field of quantum optics, which at first was so new and unfamiliar to me. So, after a year and a half of hard work, during which I got to expand my knowledge in math and physics far beyond the school curriculum, perform dozens of simulations, and for the first time in my life produce original results, I went back to work on the class yearbook and to pick out a dress for graduation. Not long afterwards, my principal suggested that I enter a competition of the Weizmann Institute and present my project. After presenting my research in Hebrew and in English, I was invited to the award ceremony, where I surprisingly found out I had won

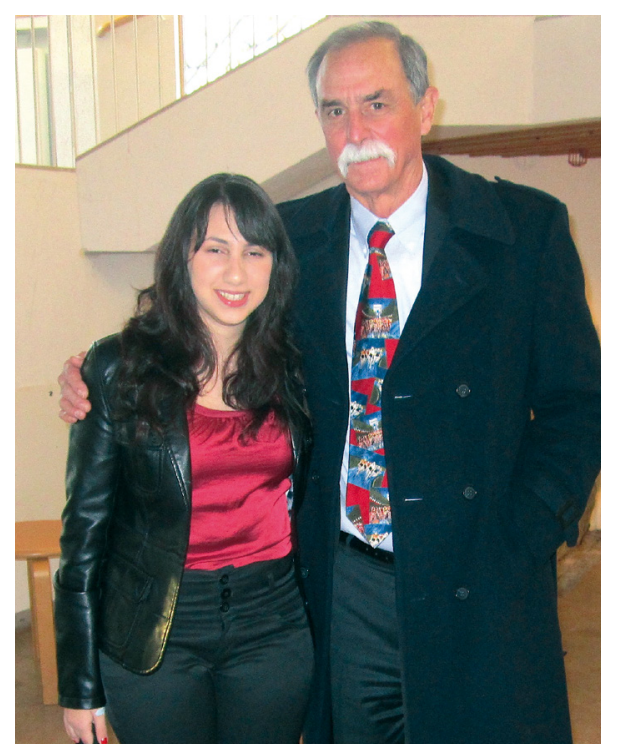

\ With David Wineland participants were a diverse group of 25 brilliant students from all over the world. People from 6 different continents had traveled for hours, even days, in order to get to Sweden for this special occasion. The one thing we all had in common was our passion for science and research, and somehow this was enough to make us become good friends by the end of the week. I'm grateful for the technology that allows me to stay in touch with those wonderful people. We went to conferences, heard and gave lectures, went sightseeing, learned about different cultures, mingled with Nobel winners, rode limos and - mostly - had a lot of fun. Personally, I think the etiquette dinner we had was most enjoyable; we were taught manners so that we would behave appropriately at the royal banquet. The guys were perfect gentlemen; we toasted and danced the waltz, feeling so classy... At the actual dinner, we used the skills we had gained while eating a fancy dish cooked by Sweden's chef of the year (or, in my case, a kosher version of it).

Then we went to the "Nobel night cap", which is the exclusive after-party of the ceremony. When looking around, it would look like just another ordinary party, with music, dancing and drinks. It is only after noticing a Nobel Prize winner passing by that you realize how great of an honor it is to be there. Furthermore, while I was there, I had the extraordinary and unique opportunity to personally meet Dr. David Wineland, 2012 Nobel the first place. I must mention that, out of the 12 students who made it to the finals of the contest, 10 were girls. Prof.Ada Yonath, 2009 Nobel laureate for chemistry, was the one who handed me the diploma, after a fascinating lecture about her research, and about how - by keeping her priorities right - she also managed to raise a wonderful family.

The main prize of the contest was representing Israel at SIYSS (Stockholm International Youth Science Seminar). It was a week full of activities associated with the Nobel Prize Award ceremony and related events. The laureate for physics, thanks to my guide, and prof. Martina Knoop, who happened to have contacts with him due to the lucky coincidence that we've all been studying the same field. Although he has achieved so much in his life, he spoke to me as an equal, and inspired me so much. In conclusion, this was an amazing experience, and I'll never forget how lucky I was to take part in it. Since I got back I've been thinking about studying abroad, and expanding my horizons as much as I can, so that one day I could make my own contribution to science just like the talented people I've met. 